\title{
Observations on the experiments of Messrs. Foucault and Fizeau, relative to the action of the red rays upon Daguerreotype plates
}

\section{Edmond Becquerel}

To cite this article: M. Edmond Becquerel (1847) Observations on the experiments of Messrs. Foucault and Fizeau, relative to the action of the red rays upon Daguerreotype plates, Philosophical Magazine Series 3, 30:200, 214-218, DOI: 10.1080/14786444708645644

To link to this article: http://dx.doi.org/10.1080/14786444708645644

曲 Published online: 30 Apr 2009.

Submit your article to this journal $₫$

Џll Article views: 4

Q View related articles $₫$ 
Our method requires the employment of iodine and bromine, and is easily performed by persons who are accustomed to employ those substances separately. It consists in polishing and iodizing the plate in the ordinary manner, and afterwards causing it, by any convenient means, to absorb three times as much vapour of bromine as is usually thought sufficient to render the plates as sensitive as possible. Whilst the ordinary quantity of bromine does not visibly alter the tint of the iodized layer, that which we recommend causes it to assume a deep bluish violet tint.

The sensihility of the plates thus surcharged with bromine is re. duced to a third of what it would be if the ordinary quantity only were used; but at the same time they are rendered capable of producing a perfect picture of subjects presenting the greatest variety of shade. This will be seen by inspecting a small picture, presented herewith, which was produced when the sun was shining. There will be perceived the clouds in the sky, white houses, with the shadows well-defined, and trees, the foliage of which is delineated much in the same manner as if executed by an artist.

We recommend the ordinary quantity of bromine to be exactly tripled; as if less than this quantity be used, the picture will not be properly brought out; and if more than this quantity were used, the mercury would not be properly condensed and the image would not be so well-defined.

This new property, communicated to the iodized plates by an excess of bromine, may be very usefully applied; and besides, as it appeared to us that this statement might be useful to the chemical world, we have been induced to make this communication to the Academy.-Comptes Rendus des Séances de l'Académie des Sciences, (as inserted in the London Journal.)

OBSERVATIONS ON THE EXPERIMENTS OF MESSRS. FOUCAULT

AND FIZEAU, RELATIVE TO THE ACTION OF THE RL'D RAYS UPON DAGUERREOTYPE PLATES. BY M. EDMOND BECQUEREL.

Messrs. Foucault and Fireau, at the sitting of the Academy on the 5th inst. (October 1846), presented some observations concerning the action of the red rays upon Daguerreotype plates, from which it would appear that the least refrangible part of the solar spectrum acts upon the iodide of silver in an inverse manner to the most refrangible portion. As it appears that these gentlemen are not acquainted with the experiments which have been made during the last few years on this subject, and as the results stated by them do not appear to me to lead to the conclusions which they deduce, I take the liberty of submitting some remarks thereon to the Acaderny.

Dr. Draper (Philosophical Magazine, Nov. 1842), on examining the image produced by the action of the spectrum upon iodized plates of silver, made known, before these gentlemen, the existence of pro. tecting rays modifying the influence of the solar rays, and even acting negatively upon the iodide of silver. Sir J. F. W. Herschel examined the pictures on that occasion, and in a very interesting experiment 
(Philosophical Magazine, Feb. 1843) on the different appearances which the iodized plates assume when exposed for the same space of time to varying intensity in the light, and submitted to the vapour of mercury, attributed these effects to the unequal thickness of the substances deposited upon the plates of silver serving as reflecting surfaces. He showed, moreover, that on operating upon paper covered with iodide of silver, nothing was observed which indicated the action of negative rays, but that all the active parts of the solar spectrum acted chemically in the same manner on the iodide.

The experiments which I have made on the chemical action of the solar rays, from 1841 to 1844 , have all led me to the same conclusion. Attention ought not therefore to be directed to the deposits formed on the surface of Daguerreotype plates, as if these were the only data to lead to the conclusion that the rays acted in various ways; for if so, there would be risk of defective results.

In support of the foregoing I will cite the following experiment, of the accuracy of which any person will be able to judge. "Let a Daguerreotype plate be prepared with iodine only (in order to avoid the admixture of active substances), and let the blue, indigo, and violet parts of a purified blue spectrum, presenting Fraunhofer's black lines, be thrown upon it. If the action only lasts a short time, after submitting it to the mercury vapour, the black lines will be seen to fix themselves upon a white ground, which represents those parts affected by the active parts of the spectrum *." But if the plate be exposed to the spectrum for an hour or more, then the appearance of the plate changes, on passing it through the mercury vapour; the lines of the spectrum are scarcely marked, and the action has been nearly uniform throughout its surface, but the lines which are visible appear white, and show very distinctly upon the ground, which approaches to blackness : the effect is quite contrary to what it was before. That portion of the plate which is acted upon by the violet part of the spectrum has, under these circumstances, the same ape pearance as the portion of the plate exposed to the red rays by Messrs. Foucault and Fizeau; and to produce this effect, it was only neces. sary to vary the time of exposure of the plate to the same portion of the spectrum. Must it be inferred, in the second case, that the iodide of silver had been acted upon by negative rays? Certainly not; for if the experiment be repeated upon iodide of silver laid upon paper, the paper will become darker and darker, in proportion to the time it continues exposed to the spectrum: and, besides, I have found (Annales de Chimie ot de Physique, 3rd series, vol. ix. p. 268 et seq:) that the electrical effects arising from the chemical decomposition of the iodide always act in the same direction.

It will be seen that the conclusion to which Messrs. Foucault and Fizeau came, viz. that there exist in the red prismatic rays negative rays, eannot be received, simply from the fact that the Daguerreo. type plates are not always the same in appearance. The contrary effects, as will be seen hereafter, are secondary effects produced by

* E. Becquerel on the Constitution of the Bolar Spectrum,-Bibliothèque Universelle de Genève, August 1842. 
several chemical reactions taking place simultaneously, and are not due to contrary action, exercised on the part of the solar rays, on the iodide of silver alone.

Another fact which I will call attention to is, that the least refrangible part of the spectrum, instead of possessing a negative action upon the iodide of silver, exercises a continuous influence upon most of the salts of silver alone, such as the iodide, the bromide, and the chloride; and also that the experiments upon which this proposition is founded have been verified by the commissioners of the Academy, charged with the examination of one of my memoirs.

In the foregoing, the only point discussed was the influence of light upon iodide of silver, or the simple salts of that metal. When plates of silver are exposed successively to the vapours of iodine, bromine or chlorine, the mixtures obtained may give rise to various kinds of chemical reaction, of which the result only is appreciable. For this reason these mixtures must not be employed without great caution, and the Daguerreotype plates must be used as little as possible in experimenting as to the nature of the active rays.

In order to show how far the mixture of sensitive materials is capable of influencing the effects of the spectrum, I will direct attention to the following observation of Sir J. Herschel :-

If paper be prepared, first with a strong solution of lead and afterwards with bromate of potash and nitrate of silver, a surface will be produced which will speedily become black on exposure to the light; on being presented to the spectrum, the black tint will be produced in the most refrangible rays, as far as green. But if the paper has been blackened by previous exposure to the light, on being covered with a dilute solution of iodide of potassium and exposed to the blue part of the spectrum, the paper will become white. This result proves that the iodide of potassium is decomposed, and that the silver which stained the paper being iodized, and coming in contact with an alkaline iodide ceases to be affected by the light; the paper will therefore remain of a yellowish-white in that portion of the spectrum on which the reaction takes place.

If the layer of iodide of potassium employed is produced from a dilute solution of that salt, the paper not only becomes white in the violet part of the spectram, but also becomes darker in the red rays, and even beyond, a neutral line being in the middle. It would appear therefore, from an examination of the image thus obtained, that two contrary results were produced; viz. the destruction of the colour in the violet part and its augmentation in the red. These effects may be easily explained as two distinct chemical reactions : first, the action of the light upon the iodide of silver, the colouring of which had commenced ; second, the action of the light to effect the decomposition of the iodide of potassium, and the iodizing of the silver arising from the sub-iodide formed by the first reaction. As the red part of the spectrum contains those rays which continue the chemical action commenced upon the salts of silver, and as the first reaction is only commenced, the latter has most influence in that part of the spectrum. The second reaction is, on the contrary, 
at its height in the violet. Thus these appearances of inverse action in the colouring do not arise from two distinct effects, positive and negative, produced by the rays upon the same sensitive surface, but are owing to two distinct chemical reactions, which predominate respectively in the red and violet parts.

If the blackened paper be covered with a fresh layer of iodide of potassium, it will begin to turn white at the least refrangible part, and the neutral line will again approach the red; if a sufficient quantity of iodide be used, the paper will turn white from the violet to the red; but if a very strong solution of iodide were employed, the paper would whiten, even in the dark; so violent is the action of the iodide of potassium upon metallic silver.

These results clearly prove that several chemical actions may take place simultaneously in the mixtures of sensitive substances, the results only of which are observable. Analogous effects must necessarily be produced on employing iodized plates of silver, and afterwards exposing them to the vapour of bromine or to chlorine; and perhaps even when using plates iodized according to M. Daguerre's plan. In fact, under these circumstances, the iodide, chloride, or bromide of silver, are in direct contact with the metallic silver; and as, by the decomposition of these salts, through the action of the light, subsalts are formed, the result is that iodine, chlorine and bromine are exposed directly to the above-named salts, and even to the metallic silver itself, at the moment when the solar action makes its influence felt. These reactions, which are sufficiently complex, become more so by the iodides, chlorides, and bromides of silver being submitted to the action of rays which always act with the same energy in the violet part of the spectrum; whilst in the red portion the rays react with greater energy, owing to certain chemical actions naving commenced.

It is therefore essential to distinguish between the chemical reactions effected under the influence of light upon sensitive substances alone, and upon combinations of them. This has not been done by Messrs. Foucault and Fizeau : they have considered a Daguerreotype plate as offering a separate sensitive surface, whilst it is only by a mixture of substances that different effects can be produced in the various parts of the spectrum, as is proved by Herschel's experiment, and without the existence of rays acting in an inverse direction. 'Thus it has been proved by experiment, that the solar rays, although of various degrees of refrangibility, only act in one way upon iodide of silver; whilst a mixture of this substance with other matters may occasion several chemical reactions acting conjointly and hiding the principal effect.

If the light acts only in one way upon iodide of silver (the continuous rays included), the case may be different on other sensitive substances, and the rays may act sometimes positively and sometimes negatively. It is known, in fact, that each sensitive substance is differently affected by the solar rays; this might be explained by stating that each sensitive substance receives the rays in a manner peculiar to itself. I will cite as an example an observation of Wol-

Phil. Mag. S. 9. Vol. 30. No. 200. March 1847. 
laston's, with regard to the sensitive substance guaiacum, which becomes blue beyond the violet part of the spectrum, and again becomes colourless in the red and yellow rays.

I have confined myself to the foregoing observations, on the subject of Messrs. Foucault and Fizeau's note, to show that the complicated phænomena produced by the appearance of the Daguerreotype plates (although very important for the photographic images produced in the camera-obscura, and for the observation of active rays of very little intensity) cannot lead to definite and unvarying results, as regards the nature of the chemical action produced. It is therefore necessary to operate, as has been heretofore done, by means of simple products, with sensitive paper, regard being had to the electrical effect due to the chemical reaction produced under the influence of the solar rays.-Ibid.

\section{RESEARCHES ON MELION AND ITS COMPOUNDS. BY MM. AUG. LAURENT AND CH. GERHARDT.}

The authors observe that in their last memoir on this subject they had not repeated the experiments of M. Liebig; that they had merely modified a part of his theory, the authority of his name appearing to be a sufficient guarantee for the accuracy of his results.

According to M. Liebig, this substance is composed of-

Six atoms of carbon $\ldots \ldots=458.61 \quad 39 \cdot 36$

Eight atoms of azote $\ldots \ldots=708.16 \quad 60.64$

One atom of mellon. . . . . . $=\overline{1166.77} \quad \overline{100 \cdot 00}$

According to him, it is a radicle composed of carbon and azote, capable of uniting with hydrogen, oxygen and metals, so as to form compounds analogous to those into the composition of which cyanogen enters.

The authors state, and they do it with regret, that the reliance which they placed on the results of M. Liebig has totally misled them; and that it is not a part only of his labours on this subject which is erroneous, but that this is the case with the entire history of mellon, with all its transformations and all its reactions. The authors assert that they are also in a condition to prove that the supposed analogy with cyanogen does not exist; for instead of consisting only of carbon and azote, mellon contains besides one and a half per cent. of hydrogen. Various processes have been employed for obtaining mellon. That analysed by MM. Laurent and Gerhardt was obtained very pure by calcining chlorinated cyanamide (cyanamide chlorée) as long as hydrochloric acid and hydrochlorate of ammonia were disengaged.

The following are the results of four experiments performed to ascertain the composition of mellon :-

\begin{tabular}{|c|c|c|c|c|}
\hline $\begin{array}{l}\text { Carbon ....... } \\
\text { Hydrogen } \ldots \ldots \\
\text { Azote ......... }\end{array}$ & $\begin{array}{r}\mathrm{I} . \\
36 \cdot 0 \\
1 \cdot 7 \\
61 \cdot 3\end{array}$ & $\begin{array}{r}\text { II. } \\
35 \cdot 8 \\
1 \cdot 8 \\
62 \cdot 4\end{array}$ & $\begin{array}{r}\text { III. } \\
36 \cdot 4 \\
1 \cdot 7 \\
61 \cdot 9\end{array}$ & $\begin{array}{r}\text { IV. } \\
35 \cdot 73 \\
1 \cdot 77 \\
62 \cdot 50\end{array}$ \\
\hline & 99.0 & $100 \cdot 0$ & $100 \cdot 0$ & 100.00 \\
\hline
\end{tabular}

